\title{
Complicações das rinossinusites
}

\author{
Felipe Marconato,, ${ }^{1,3 *}$ João Mangussi-Gomes, ${ }^{1,2}$ Leonardo Balsalobre ${ }^{1,2}$
}

\section{Resumo}

Introdução: As complicações das rinossinusites (RS) incluem uma série de doenças que necessitam de reconhecimento e tratamento imediatos. A literatura descreve várias formas de classificação e definição destas doenças. Objetivos: Descrever aspectos epidemiológicos e fisiopatológicos das complicações das rinossinusites. Métodos: Revisão narrativa utilizando artigos recentes e históricos que norteiam nossa conduta e são utilizados como base dos tratamentos atuais. Resultados: Dentre as complicações das RS, as que mais se destacam pela frequência são as orbitárias, responsáveis por até 75\% dos casos, principalmente em crianças. Além das orbitárias, podem ocorrer ainda as complicações ósseas e cerebrais, constituindo um grande desafio, tanto pela sua dificuldade diagnóstica, como pelos altos índices de morbimortalidade. Conclusão: Independente do tipo de complicação, deve-se sempre ter em mente esses diagnósticos diferenciais a fim de se iniciar o tratamento específico o mais precocemente possível e evitar desfechos desfavoráveis.

Descritores: Complicações; Sinusite; Abscesso; Celulite orbitária; Empiema subdural.

\section{Abstract \\ Rhinosinusitis complications \\ Introduction: The rhinosinusitis (RS) complications inclu- de a range of diseases that require immediate recognition and treatment. The literature describes several ways of classifying and defining them. Objectives: To describe epidemiologic and fisiologic aspects of the RS complica- tions. Method: Narrative review using recent and historical articles that guide our conduct and are the basis of current treatments. Results: Among the complications of RS, the most frequent are the orbital, responsible for $75 \%$ of cases, mainly in children. Besides the orbital, there are bone and brain complications, constituting a great challenge, as much for diagnostic difficulty as for high morbidity and mortality rates. Conclusions: Despite the complication, it is important to keep in mind these differential diagnoses in order to provide the specific treatment soon as possible and avoid unfavorable outcomes.}

Keywords: Complications; Sinusitis; Abscess; Orbital cellulitis; Empyema, Subdural.
1. Centro de Otorrinolaringologia e Fonoaudiologia (COF). Complexo Hospitalar Edmundo Vasconcelos (CHEV). São Paulo, SP, Brasil.

2. Departamento de Otorrinolaringologia e Cirurgia de Cabeça e Pescoço. Universidade Federal de São Paulo (UNIFESP). São Paulo$\mathrm{SP}$, Brasil.

3. Núcleo de Medicina Avançada. Hospital Sírio Libanês. São Paulo, $\mathrm{SP}$, Brasil.

\section{*Endereço para correspondência:}

Rua Afonso Brás, 525, CJ 13

São Paulo, SP, Brasil. CEP: 045119-02.

E-mail: felipemarconato.orl@gmail.com

Revista HUPE, Rio de Janeiro, 2016;15(4):349-355

doi: 10.12957/rhupe.2016.31616

Recebido em 18/03/2017. Aprovado em 24/11/2017.

\section{Resumen}

\section{Complicaciones de la rinosinusitis}

Introducción: Las complicaciones de la rinosinusitis (RS) son una serie de dolencias que requienen un reconocimiento y tratamiento inmediato. La literatura describe varias formas de clasificación y de definición de ellas. Objetivos: Describir aspectos epidemiológicos y fisiopatológicos de las complicaciones de la RS. Método: Revisión narrativa utilizando artículos recientes e históricos que orientan nuestra conducta y se utilizan como base de los tratamientos actuales. Resultados: De entre las complicaciones de las RS, las que más destacan por su frecuencia son los orbitales, responsables por el $75 \%$ de los casos, principalmente en niños. Más allá de la órbita, también pueden ocurrir complicaciones óseas y cerebrales, siendo éstas un gran desafío, tanto por sus dificultades diagnósticas, como por los altos índices de morbilidad y mortalidad. Conclusiónes: Independientemente del tipo de complicación, hay que tener en mente estos diagnósticos diferenciales con el objetivo de iniciar un tratamiento específico lo más precozmente posible y así evitar resultados desfavorables.

Palabras clave: Complicaciones; Sinusitis; Absceso; Celulitis orbitaria; Empiema subdural. 


\section{Artigo de revisão}

\section{Introdução}

As complicações das rinossinusites (RS) envolvem um conjunto de doenças inflamatórias/infecciosas que se estendem para além dos limites do nariz e dos seios paranasais. São condições que devem ser prontamente identificadas e tratadas pois o atraso no seu manejo pode resultar em elevada morbimortalidade. ${ }^{1} \mathrm{Os}$ objetivos do presente estudo foram descrever aspectos epidemiológicos e fisiopatológicos das complicações das rinossinusites.

\section{Epidemiologia}

Estima-se que a incidência anual de complicações de RS seja de aproximadamente 3 casos para cada $1 \mathrm{mi}-$ lhão de habitantes ao ano. Apesar disso, esses números podem variar de acordo com a região, o sexo, a faixa etária, o tipo de complicação e o tipo de RS estudada (se aguda ou crônica, por exemplo). Assim, as complicações de RS são mais frequentes em pacientes do sexo masculino (proporção de 2,6 homens para cada 1 mulher) e em crianças - estima-se que ocorra 1 complicação para cada 12.000 casos de RS em crianças, enquanto para adultos esse número é de 1 complicação para cada 32.000 casos. Além disso, em crianças, as complicações geralmente advêm de um processo agudo (rinossinusite aguda RSA), ao passo que, em adultos, as complicações estão mais comumente associadas a episódios de rinossinusite crônica (RSC). ${ }^{1,14}$

A evolução da antibioticoterapia ocorrida nas últimas décadas reduziu drasticamente a morbimortalidade associada às complicações das RS. Entretanto, é interessante observar que não houve nenhuma alteração na incidência das complicações das RS. O uso excessivo de antibióticos, na verdade, eleva a taxa de resistência bacteriana e pode, ainda, mascarar muitas complicações das RS, o que contraindica seu uso indiscriminado., ${ }^{2,4}$

Conforme o local acometido, as complicações das RS podem ser divididas de acordo com a lista a seguir, com respectivas frequências:

- Orbitárias: 60-75\%

- Intracranianas: $15-20 \%$

- Ósseas: 5-10\%

As complicações orbitárias são as mais comuns e predominam em crianças, enquanto as intracranianas e ósseas são mais frequentes em adultos. ${ }^{1,5,13,16}$

\section{Fisiopatologia}

Várias teorias tentam explicar o motivo da ocorrência das complicações das RS. Sabe-se que a disseminação da inflamação e da infecção ocorre, principalmente, por mecanismos de continuidade ou contiguidade. Algumas características anatômicas específicas do narize dos seios paranasais facilitam e justificam esses processos:

- o limite anatômico entre as células etmoidais e a órbita é composto pela lâmina papirácea, uma fina camada de osso;

- além de delgada, a lâmina papirácea contém forames neurovasculares relativamente mais largos e suturas ósseas não completamente fechadas, principalmente em crianças, quando comparada a outras estruturas ósseas;

- $\quad$ podem existir defeitos ósseos, adquiridos ou congênitos, na lâmina papirácea e nos outros limites ósseos entre o nariz, os seios paranasais e as estruturas adjacentes.

Ainda, a existência de um sistema venoso avalvular (exemplificado pelas veias etmoidais, oftálmicas e pelas veias da díploe do osso frontal - chamadas veias de Breschet) justifica a disseminação do processo inflamatório por via hematogênica, de maneira retrógrada. ${ }^{1,6-18}$

\section{Classificação}

Existem várias classificações propostas para as complicações de RS. A classificação mais aceita e utilizada, ainda nos dias de hoje, é a proposta por Chandler, em 1970. ${ }^{6}$ Essa classificação leva em consideração o septo orbitário, formado por uma deflexão anterior do periósteo da órbita, considerado uma importante barreira anatômica para propagação das infecções. ${ }^{6,15}$ De acordo com essa classificação, podemos dividir as complicações em: ${ }^{6}$

o Pré-septais:

- Grupo 1 - Celulite/Edema peri-orbitário

o Pós-septais:

- Grupo 2 - Celulite orbitária

- Grupo 3 - Abscesso subperiosteal

- Grupo 4 - Abscesso orbitário

- Grupo 5 - Trombose de seio cavernoso

Com base em achados da tomografia computadorizada (TC) e da ressonância magnética (RM), Mortimore $\&$ Wormald(1997), por sua vez, classificaram a trombose do seio cavernoso entre as complicações intracranianas e não mais entre as orbitárias, reduzindo a classificação para 3 grupos: ${ }^{7}$

- Grupo 1 - Infecção pré-septal

- Grupo 2 - Infecção pós-septal subperiosteal

- Grupo 3 - Infecção pós-septal intraconal

Os 3 grupos podem ainda ser subdivididos em " $\mathrm{A}$ " (edema/inflamação) ou "B" (abscesso). 
No Brasil, Velasco e Cruz e colaboradores propuseram uma classificação mais simples e lógica, em que não foram incluídas as complicações pré-septais, nem as intracranianas: ${ }^{8}$

- Grupo 1 - Celulite orbitária

- Grupo 2 - Abscesso subperiosteal

- Grupo 3 - Abscesso orbitário

\section{Complicações orbitárias}

É o grupo mais comum de complicações das RS e acomete principalmente crianças. O principal sítio de origem são as células etmoidais, seguido pelos seios maxilares e frontais.

O diagnóstico das complicações orbitárias é clínico e radiológico. A acurácia do diagnóstico clínico é de cerca de $82 \%$ e aumenta para 91\% quando associado à tomografia computadorizada (TC). ${ }^{1} \mathrm{~A}$ TC com contraste é o principal exame a ser pedido, pela capacidade de identificar estruturas ósseas, áreas de inflamação e abscessos. A ressonância magnética (RM), por sua vez,é muito útil para a avaliação do acometimento de partes moles, principalmente para as complicações pós-septais e intraconais. ${ }^{5}$

A celulite periorbitária é a complicação orbitária mais comum. É caracterizada por edema, eritema e dor palpebral, sem alteração da acuidade ou mobilidade ocular. Menos de 10\% dos casos de celulite periorbitária são devidos a episódios de RS, sendo mais comumente causados por infecções cutâneas, oftalmológicas ou virais de vias aéreas superiores. O diagnóstico é eminentemente clínico e o tratamento pode ser feito, na maior parte dos casos, de forma domiciliar, com antibiótico via oral. ${ }^{9}$ Apesar disso, deve-se manter acompanhamento próximo pois o quadro pode evoluir para abscesso palpebral ou até mesmo para complicações pós-septais. ${ }^{1,5,8}$

A celulite orbitária ocorre quando o processo inflamatório/infecciso invade a região pós septal e envolve a gordura orbital. É caracterizada por exoftalmia, quemose e hiperemia de conjuntiva, geralmente preservando a mobilidade ocular e sem alteração da acuidade visual, podendo estar acompanha de sintomas sistêmicos. ${ }^{1}$ Torna-se necessária a TC para diagnóstico e o tratamento deve ser imediato com antibiótico endovenoso. A não melhora do quadro clínico com o tratamento, a evidência de abcesso na TC e a piora da visão indicam a cirurgia para drenagem e exploração orbitária. $^{5}$

O abscesso subperiosteal é caracterizado por um quadro clínico mais marcante, com exoftalmia, exoforia, alteração da mobilidade ocular, dor à movimentação ocular, redução da acuidade visual e sintomas sistêmicos. Os abscessos localizados na região medial da órbita são os mais frequentes (encontrados em aproximadamente $58 \%$ dos casos) e geralmente originam-se de sinusite das células etmoidais. Os abscessos superiores, inferiores e laterais são mais raros, com frequências estimadas em 21\%, 13\% e 8\%, respectivamente. ${ }^{10}$ A TC com contraste normalmente mostra o abscesso bem definido, na região subperiosteal da órbita, com formado em cunha. Habitualmente, apresenta um centro hipointenso com captação periférica de contraste. $\mathrm{O}$ tratamento padrão nesses casos é a drenagem cirúrgica associada a antibioticoterapia endovenosa.

Há situações em que o abscesso subperiosteal (Figura 1) pode ser manejado de forma conservadora, sem cirurgia num primeiro momento. Essa conduta é aceitável para crianças com até 4 anos de idade, que

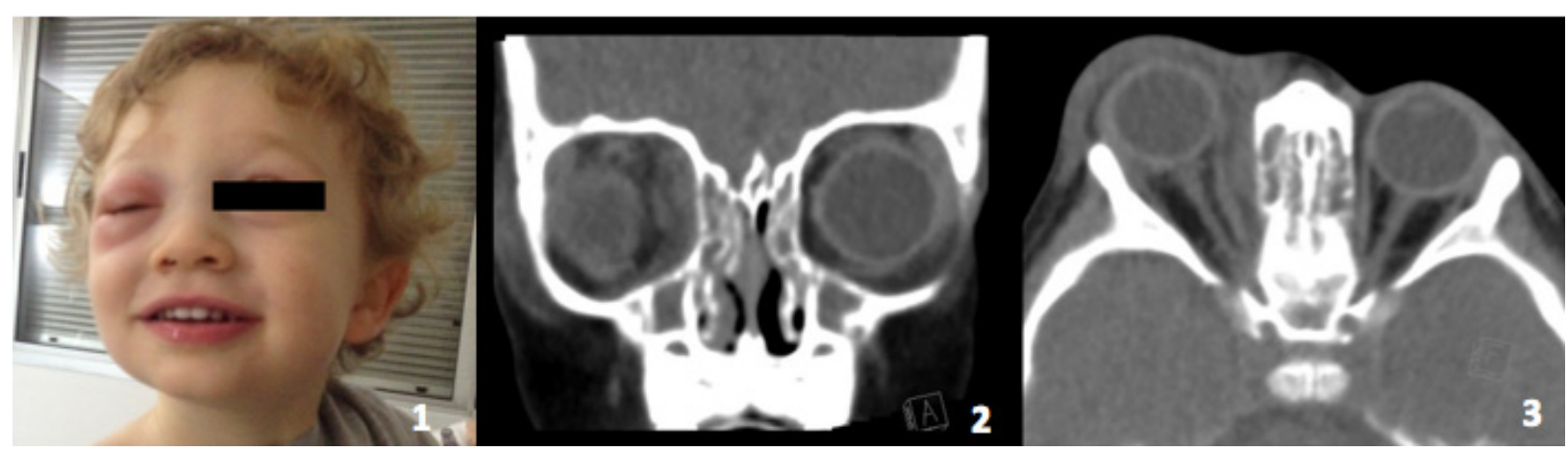

Figura 1. Tomografia de quadro de obstrução nasal e rinorreia há uma semana.

CFS, 5 anos, apresentando quadro de obstrução nasal e rinorreia há uma semana. Há dois dias evoluiu com edema e hiperemia palpebral (1). A TC em corte coronal(2) e transversal(3) mostrando quadro de sinusite etmoidal e maxilar bilateral abcesso subperiosteal medial à direita caracterizado como uma massa circunscrita, hipodensa, em parede medial da órbita e com deslocamento medial do músculo reto medial. 
apresentem coleções pequenas $(<0,5-1,0 \mathrm{~mL})$ e de localização medial, sem perda visual ou outros sinais de comprometimento ocular e sem comprometimento sistêmico significativo. Nesses casos, inicia-se antibioticoterapia endovenosa e espera-se melhora clínica significativa nas primeiras $24-48$ horas (Figura 2). Caso a melhora não ocorra nesse período, ou caso haja piora do quadro, deve-se proceder à abordagem cirúrgica do abscesso. ${ }^{1,5,16}$

Abscessos orbitários são lesões intraconais caracterizadas por exoftalmia irredutível e dolorosa, com importante prejuízo da acuidade visual. O aumento da pressão orbitária pode ocluir a artéria retiniana e, caso essa compressão dure mais do que 90 minutos, levar a degeneração definitiva da retina e do nervo, ocasionando amaurose definitiva. ${ }^{1}$ A TC mostra formação hipodensa com captação periférica de contraste em topografia intraconal; o tratamento deve ser imediato com antibiótico endovenoso. Quando são encontrados abscessos orbitários, a cirurgia e exploração orbitária estão indicados de imediato. ${ }^{5}$

Uma complicação típica ocorre quando há compressão dos nervos cranianos que atravessam a fissura orbitária superior, geralmente decorrente de uma sinusite etmoidal posterior ou esfenoidal. Caracteriza-se por envolver o III, IV e VI nervos e o ramo oftálmico do V nervo (V1), resultando em um quadro de hipoestesia e imobilidade ocular, pupilas dilatadas não fotorreagentes e ptose palpebral, conhecido como síndrome da fissura orbitária superior. Quando junto com essas estruturas houver comprometimento do conteúdo do forame óptico, haverá amaurose e dor ocular intensa, quadro que caracteriza a síndrome do ápice orbitário., 5,18

Para todas as complicações orbitárias pós-septais de RS, nos exames laboratoriais, é possível encontrar leucocitose com desvio à esquerda. Altos títulos de proteína-C-reativa (PCR), tem relação com desfechos mais graves. A bacteriologia dessas complicações é a mesma das RSA, em sua grande maioria, e são frequentemente encontrados: S. Pneumoniae, H. Influenzae, S. aureus e aneróbios (Prevotella, porphyromanas, fusobacterium e Peptostreptococcus spp). ${ }^{18}$

\section{Complicações intracranianas}

As complicações intracranianas decorrem principalmente de RS dos seios frontal e esfenoidal.Sua maior incidência está entre adolescentes e adultos jovens, principalmente do sexo masculino, ${ }^{13}$ sendo rara em crianças (pois nesses pacientes o seio frontal é pouco desenvolvido). ${ }^{14}$ Com frequência são diagnosticadas tardiamente. Do ponto de vista microbiológico, há alta incidência de anaeróbios e flora mista, presente em 50\% dos casos: S. millieri e S. anginosus, fusobacterium e S. Aureus. ${ }^{1}$

Meningite - geralmente tem origem nos seios esfenoidal, etmoidal e/ou frontal. Clinicamente se manifesta por febre, cefaleia intensa e rigidez de nuca. A TC identifica possíveis complicações adicionais e os seios acometidos. À punção lombar, realizada somente após ser excluída a possibilidade de hipertensão intracraniana por efeito de massa, é detectado o aumento do número de células e proteínas. O tratamento é clínico, com antibioticoterapia endovenosa, e a mortalidade gira em torno de 5\%.11

Trombose do seio cavernoso - caracteriza-se clinicamente por exoftalmia, ptose, paralisia do III e IV e VI nervos cranianos, anestesia corneana, dor retro-orbitária, abolição do reflexo oculomotor e papiledema,

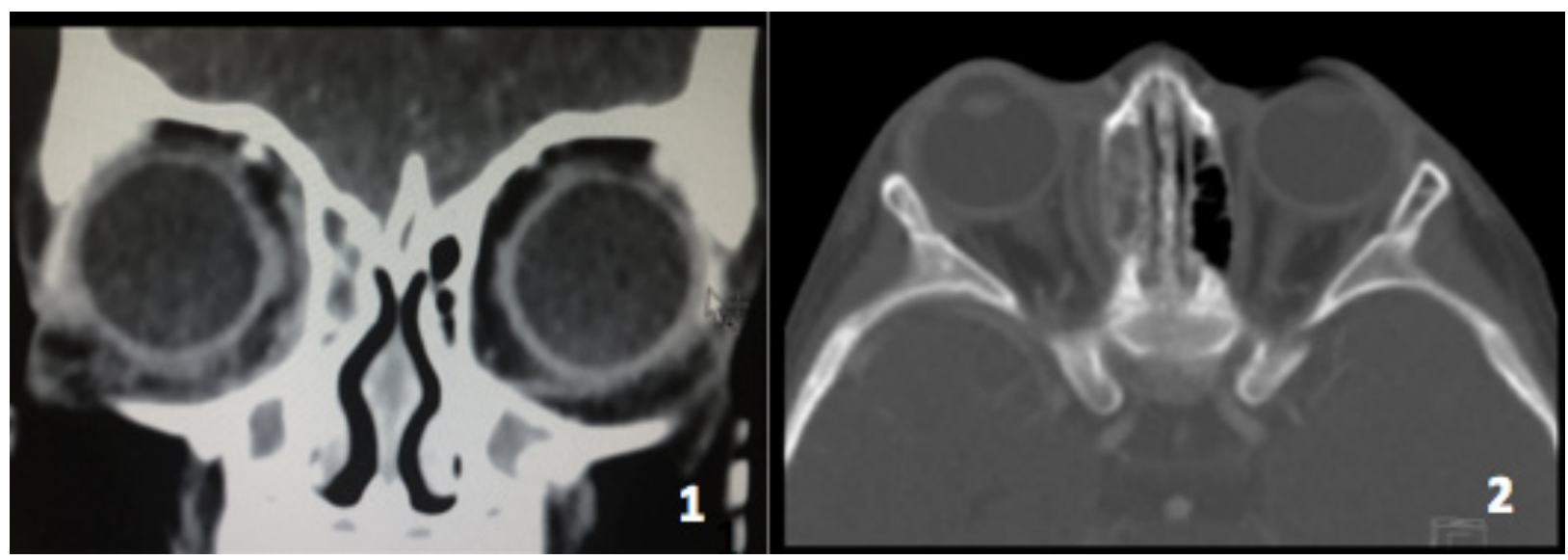

Figura 2. Tomografia após 2 semanas de tratamento clínico mostrando grande redução do abcesso subperiosteal., ${ }^{4,5}$ 
podendo estar associada a sinais meníngeos, febre e prostração. ${ }^{15,18}$ Devido à comunicação venosa que existe entre os dois seios cavernosos, os sintomas e sinais podem ser bilaterais. É uma complicação de mortalidade alta, em torno de 30\% dos casos. Normalmente, a situação exige internação hospitalar e antibioticoterapia endovenosa prolongada. A drenagem cirúrgica do seio acometido, geralmente o esfenoidal, também está indicada. O uso de anticoagulantes orais ou parenterais é controverso. ${ }^{1}$

Abscesso extradural/epidural - definido como coleção purulenta acumulada entre a dura-máter e a calota craniana, em geral associado a osteomielite do osso frontal. Tem sintomatologia discreta, que se intensifica quando ocorre efeito de massa. Pode ocorrer cefaleia, febre e alteração do comportamento, além de outros sinais centrais localizatórios. A TC que consta na Figura 3 mostra uma coleção purulenta de aspecto em lenticular, sem cruzar a linha média.,11

Abscesso subdural - é o acúmulo de pus entre a dura-máter a e pia-máter.É considerada por muitos autores como a complicação intracraniana mais comum. Apresenta sintomas neurológicos marcantes, como cefaleia intensa, febre e redução do nível de consciência. A TC mostra uma lesão em aspecto de crescente, que não respeita a linha média., 1,3,14

Abscesso cerebral e cerebrite - ocorre mais fre- quentemente na região do lobo frontal. O diagnostico geralmente é tardio, percebido somente quando advém febre, sinais de hipertensão intracraniana, crise convulsiva, alterações do nível de consciência e/ou sinais neurológicos focais. A TC mostra uma lesão arredondada com centro hipodenso e realce periférico com hipercaptação de contraste, inicialmente irregular tornando-se mais definido com a evolução do quadro. Pode ser uni ou multilocular. A punção lombar, nesse caso, está contraindicada devido risco de herniação cerebral. O tratamento habitualmente é clínico, associado a drenagem cirúrgica dos focos de supuração. 1,3,5,12,13

\section{Complicações ósseas}

Provêm mais comumente dos seios frontal e maxilar. Na região frontal, ocorre principalmente em jovens, por terem díploe abundante - camada de osso esponjoso rico em veias avalvulares entre as trabéculas ósseas interna e externa do frontal. Por essa característica, é possível o livre trânsito de sangue entre a mucosa do seio e os seios venosos do crânio.

Uma forma peculiar de lesão na região frontal é o tumor de Pott (Figuras 4 e 5), definido como uma massa amolecida, sem sinais flogísticos, resultado de um abscesso subperiosteal associado a osteomielite do seio e osso frontais. Estima-se que $40 \%$ dos casos de tumor de Pott tenham componentes orbitários ou

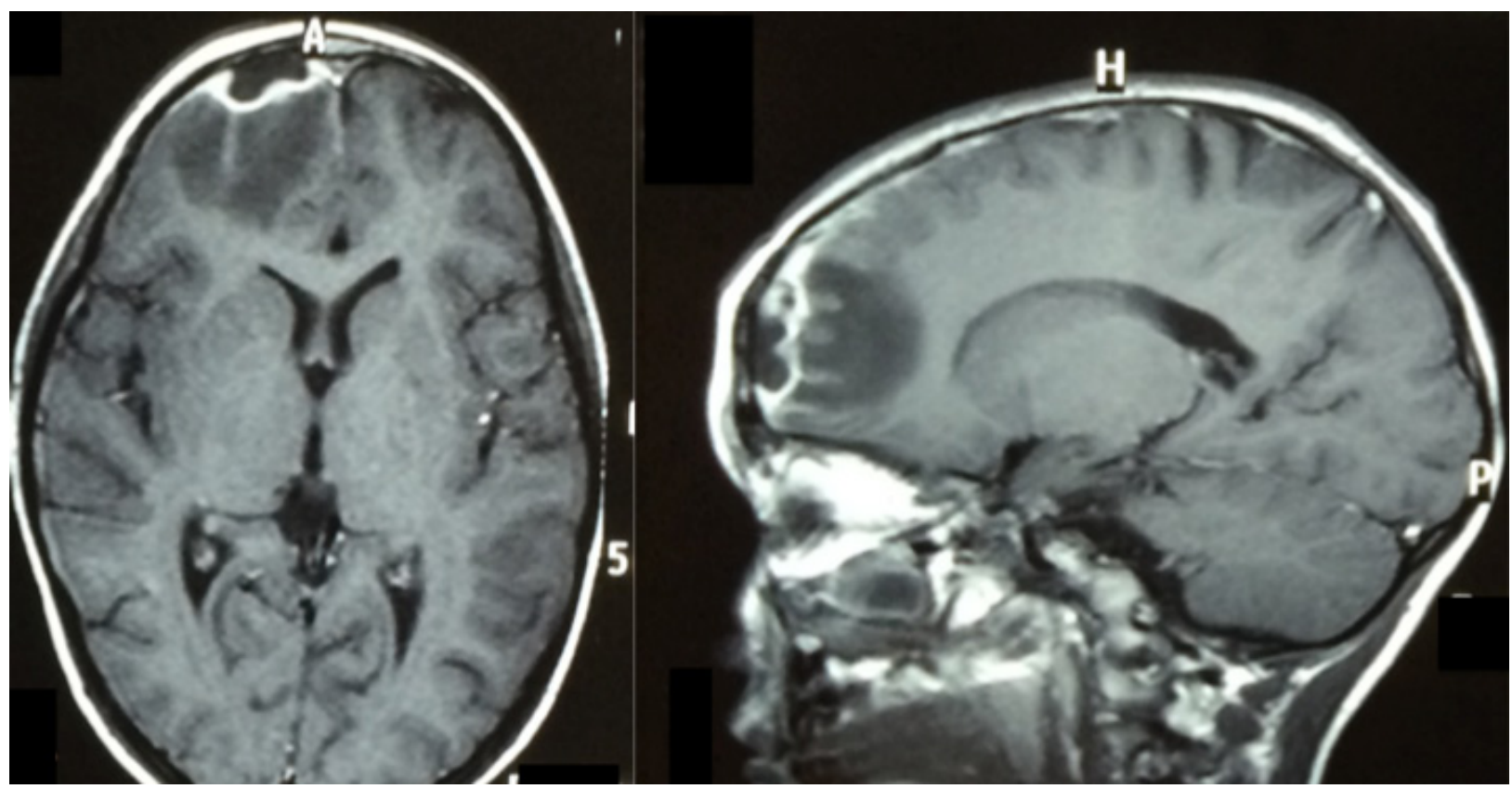

Figura 3. Abcesso epidural caracterizado por lesão entre a dura e a tábua do crânio, com realce periférico de contraste, em formato lenticular e respeitando a linha média. 


\section{$1 \mathrm{HPE}_{\text {PEDOE ENESTO }}$}

\section{Artigo de revisão}

intracranianos associados. A TC confirma o diagnóstico e a cintilografia com tecnécio pode ajudar nas fases iniciais. OStreptococcus milleri é o agente infeccioso mais comum, presente em metade dos casos. O tratamento se dá com antibioticoterapia prologada e desbridamento do osso doente.

A osteomilete do maxilar é quase sempre de causa odontogênica e mais frequente em lactentes. . $^{1,517}$

\section{Referências}

1. Fokkens WJ, Lund VJ, Mullol J, et al. EPOS 2012: European position paper on rhinosinusitis and nasal polyps 2012. A summary for otorhinolaryngologists. Rhinology. 2012 Mar;50(1):112. doi: 10.4193/Rhino50E2

2. Hansen FS, Hoffmans R, Georgalas C, et al. Complications of acute rhinosinusitis in The Netherlands. Fam Pract. 2012 Apr;29(2):147-53. doi: 10.1093/fampra/cmr062.

3. Clayman GL, Adams GL, Paugh DR, Koopmann CF. Intracranial

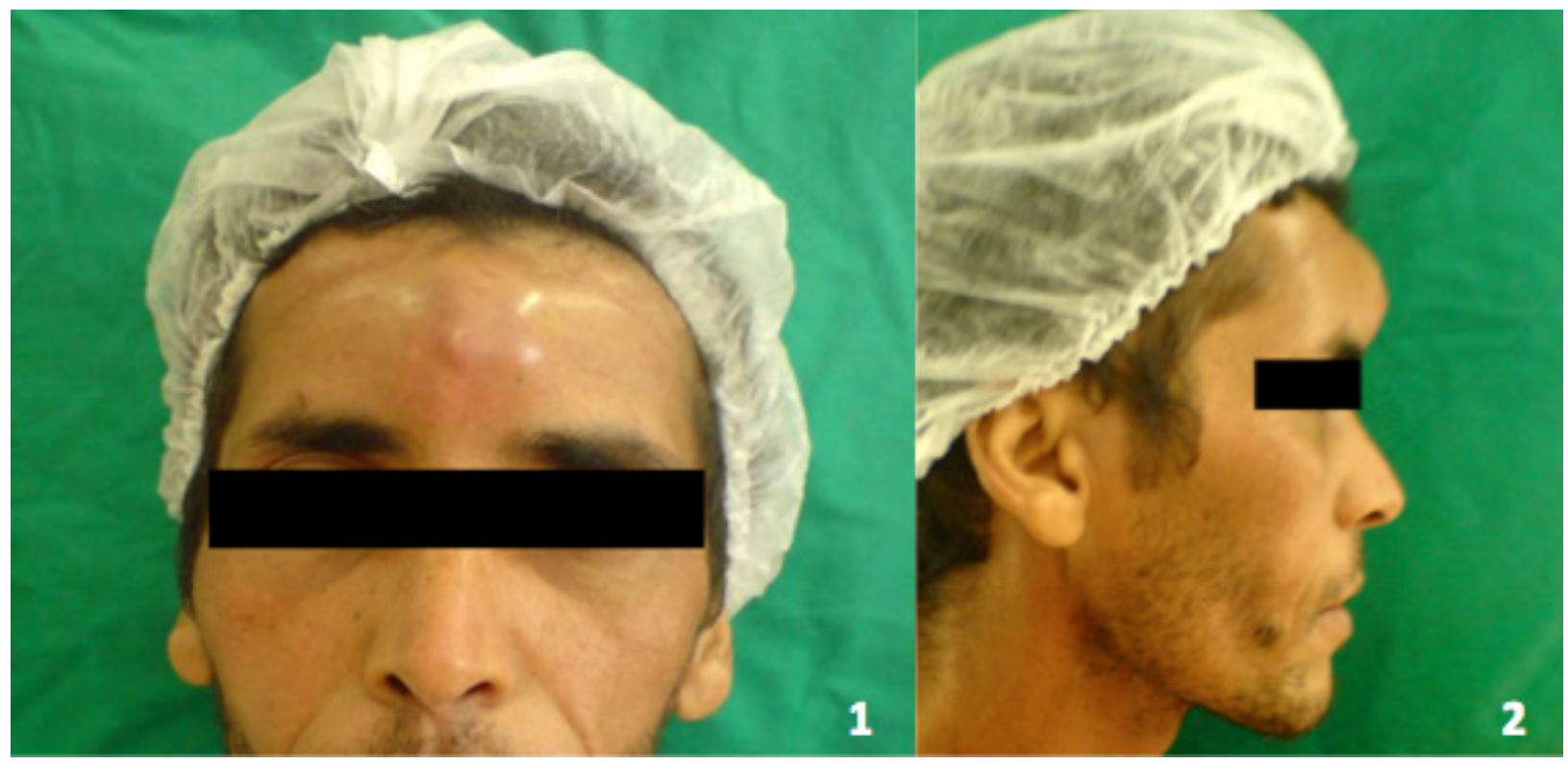

Figura 4. SVS, 35 anos com clinica de tumor amolecido em fronte há 1 semana.,

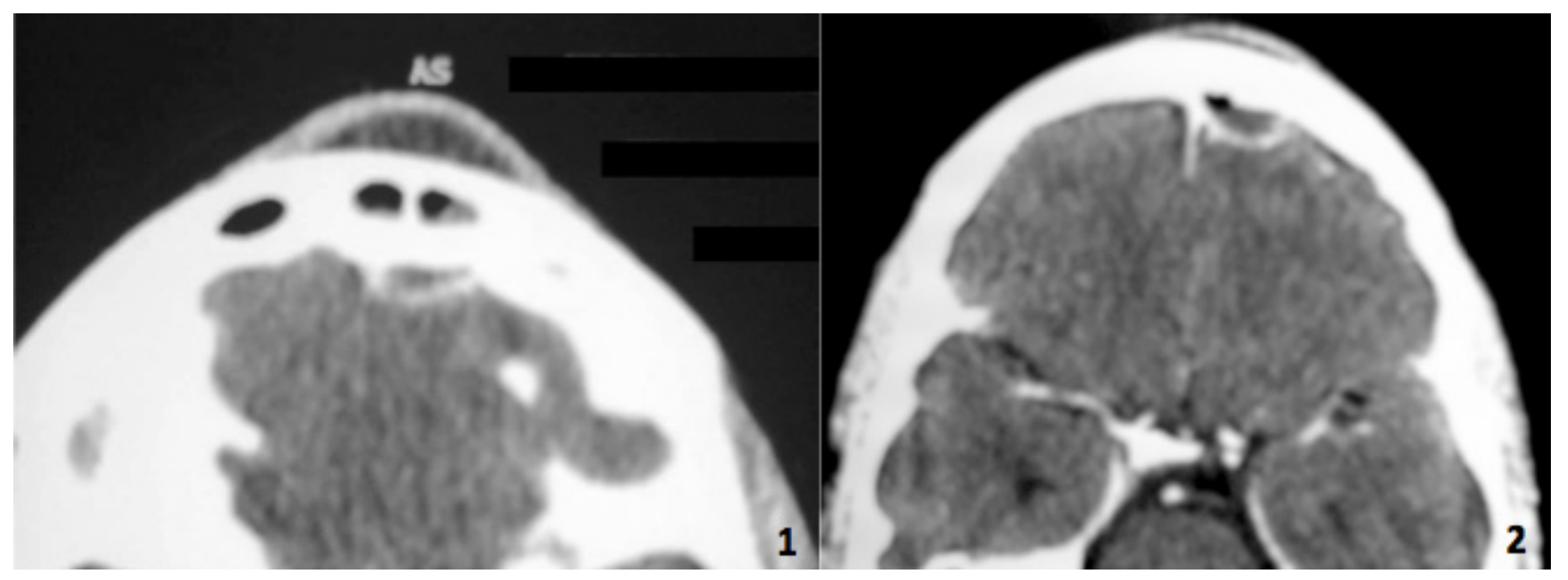

Figura 5. A TC apresenta sinusite frontal esquerda associada a massa em região frontal em topografia de subcutâneo com consistência partes moles com realce periférico pós contraste (1.2) lesão caracterizada como tumor de Pott. 
Felipe Marconato e cols. • Complicações das rinossinusites

complications of paranasal sinusitis: a combined institutional review. The Laryngoscope. 1991 Mar;101(3):234-9.

4. Jones NS, Walker JL, Bassi S, Jones T, et al. The intracranial complications of rhinosinusitis: can they be prevented? The Laryngoscope. 2002 Jan;112(1):59-63. doi: 10. $1155.2017 / 8743828$

5. Anselmo-Lima WT, Sakano E, Anselmo-Lima WT, et al. Rhinosinusitis: evidence and experience. Braz J Otorhinolaryngol. 2015;81(1):1-49. doi: 10.1016/j.bjorl.2014.11.005

6. Chandler JR, Langenbrunner DJ, Stevens ER. The pathogenesis of orbital complications in acute sinusitis. The Laryngoscope. 1970 Sep;80(9):1414-28.

7. Mortimore S, Wormald PJ. The Groote Schuur hospital classification of the orbital complications of sinusitis. J Laryngol Otol. 1997 Aug;111(8):719-23.

8. Cruz AAV, Demarco RC, Valera FCP, et al. Orbital complications of acute rhinosinusitis: a new classification. Rev Bras Otorrinolaringol. 2007 Oct; 73(5):684-8.

9. Dunham ME. New light on sinusitis. Contemp Pediatr. 1994 Oct;11(10):102-6, 108, 110 passim.

10. Hoxworth JM, Glastonbury CM. Orbital and intracranial complications of acute sinusitis. Neuroimaging Clin N Am. 2010 Nov;20(4):511-26. doi: 10.1016/j.nic.2010.07.004.

11. Younis RT, Lazar RH, Anand VK. Intracranial complications of sinusitis: a 15-year review of 39 cases. Ear Nose Throat J. 2002 Sep;81(9):636-8, 640-2, 644.
12. Germiller JA, Monin DL, Sparano AM, et al. Intracranial complications of sinusitis in children and adolescents and their outcomes. Arch Otolaryngol Head Neck Surg. 2006 Sep;132(9):969-76.

13. Phillip Hong, Charles A Pereyra, Uta Guo, et al. Evaluating Complications of Chronic Sinusitis. Case Reports in Emergency Medicine. 2017, Article ID 8743828, 3 pages. doi: 10.1155/2017/8743828

14. Neha A, Patel A, David Garber B, et al. Systematic review and case report: Intracranial complications of pediatric sinusitis. International Journal of Pediatric Otorhinolaryngology. 2016;86:200-212 . doi: 10.1016/j.ijporl.2016.05.009

15. Tara F Carr. Complications of sinusitis. American Journal of Rhinology \& Allergy. 2016;30(4):241-245. doi: 10.2500/ ajra.2016.30.4322

16. Teinzer F, Stammberger H,Tomazic PV. Transnasal Endoscopic Treatment of Orbital Complications of AcuteSinusitis: The Graz Concept. Ann Otol Rhinol Laryngol. 2015 May;124(5):368-73. doi: $10.1177 / 0003489414558110$

17. Domville-Lewis C, Friedland PL, Santa Maria PL. Pott's puffy tumour and intracranial complications of frontal sinusitis in pregnancy. J Laryngol Otol. 2013 Jan;127 Suppl 1:S35-8. doi; 10.1017/S0022215112001673

18. Atherino CCTA, Meirelles RC. Complicações das Rinossinusites. Tratado de Otorrinolaringologia volume III, ABORL-CCF. Ed. Roca; 2o Ed. 2011. 